\title{
General Anesthetic Management for Patient with Brugada Syndrome: A Case Report
}

\author{
Ji Won Bak, Se Jin Kim, Yeon JiRoh, So Yeon Cho, Seongsik Kang* \\ Department of Anesthesiology and Pain Medicine, Institute of Medical Sciences \\ Kangwon National University Hospital, School of Medicine \\ Chuncheon, South Korea \\ Corresponding author's email: sskang [AT] kangwon.ac.kr
}

\begin{abstract}
Brugada syndrome is an arrhythmogenic cardiopathy characterized by electrocardiography (ECG) pattern of the presence of an atypical right bundle branch block pattern with ST segment elevation in the precordial leads (V1-V3). It is sometimes a ssociated with sudden deaths caused by ventricular arrhythmia. Here, we are reporting a case of a 43-year-old male patient with Brugada syndrome who undenwent a tonsillectomy under general anesthesia without any complications.
\end{abstract}

Keywords --- Brugada syndrome, Cardiomy opathy, General anesthesia, Tonsillectomy, Ventricular arrhythmia

\section{INTRODUCTION}

Brugada syndrome (BS) was first described in 1992 and is a rare genetic disorder affecting sodiumchannels of the heart. It can trigger the development of malignant ventricular arrhythmias and be the leading cause of cardiac death under the age of 40 years. The diagnosis is made by ECG and defined by RBBB pattern with a characteris tic cove-shaped ST elevation in leads V1 to V3, without obvious structural heart disease. The patients with theECG pattern are susceptible to ventricular arrhythmias that may cause syncope, chest pain, or sudden death [1,2].

The reported cases of anesthetic management of the patients with BS are not abundant owing to the low prevalence of the disease [3]. Therefore, we report the case of anesthetic management for a patient with BS for tonsillectomy under general anesthesia.

\section{CASE}

A 43-year-old male patient had suffered from chronic tonsillitis and was scheduled for an elective tonsillectomy. Four months before the surgery, he was incidentally diagnosed as a Brugada syndrome on a regular medical check-up. He had no history of chest pain, syncope, and sudden cardiac arrest. Moreover, family history was negative for sudden death. ECG of the patient showed a gradually descending ST elevation above the baseline followed by a biphasic T wave resulting in a saddle-back configuration, type 2ECG Brugada pattern, in the precordial area (Figure 1). The conversion to type 1 ECG pattern was observed after provocation test with a sodium channel blocker, so he was diagnosed as asymptomatic Brugada syndrome. His cardiac enzymes were not elevated and coronary angio graphy (CAG) and coronary computed tomography angiography (CCTA) showed no abnormality of cardiovascular structures. Before the surgery, his cardiologist did not consider that there was an indication for an implanted cardioverter defibrillator(ICD) ins ertion. On preoperative evaluation, the chest radiograph and laboratory find ings including electrolytes were normal. Having been informed of the as sociated risks, the patient was taken to the operating roomand routine monitors were applied. He was $168 \mathrm{~cm}$ tall and weighed 70 $\mathrm{kg}$. Heart rate was $55 / \mathrm{min}$, non-invasive blood pressure was $127 / 66 \mathrm{~mm} \mathrm{Hg}$ and $\mathrm{SpO}_{2}$ was $97 \%$. A radialarterial cannula was inserted under local anesthesia on right radial artery. Prior to the induction of anesthesia, external defibrillator pads were attached to his anterior chest. Anesthesia was induced by fentanyl $100 \mu \mathrm{g}$, thiopental sodium $250 \mathrm{mg}$, and rocuronium $40 \mathrm{mg}$ intravenously, and maintained with sevoflurane 1.5 to $2.0 \mathrm{vol} \%$. The anesthetic depth was controlled by monitoring bispectral index (BIS) between 40 and 60. Nasopharyngeal temperature probe was placed for monitoring the core temperature. Anesthesia was terminated after 25 minutes of the surgery, and sugammdex $200 \mathrm{mg}$ was administered intravenously to reverse the neuromuscular blockade. There was no significant difference in vital signs and ECG after sugammadex administration. After the patient was physiologically stable with sufficient spontaneous breathing and $99 \%$ of $\mathrm{SpO}_{2}$, he was extubated. He was transferred to the intensive care unit for 24 hours after the operation and there was no epis ode of dysrhythmia throughout his hospital stay. 


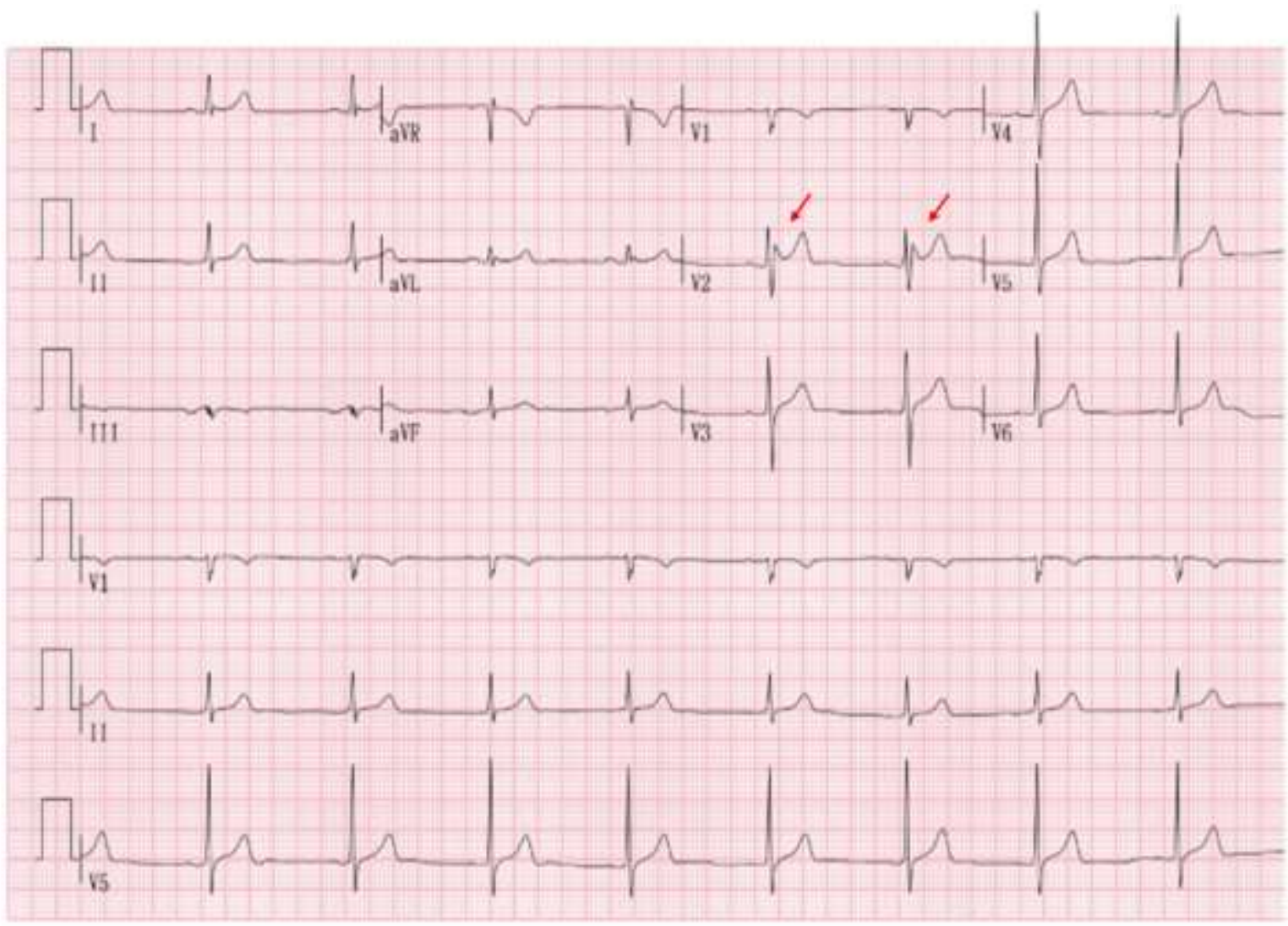

Figure 1. Preoperative electrocard iogram(ECG) of the patient. TheECG shows the saddle-back pattern in right precordial lead V2(arrow).

\section{DISCUSSION}

Brugada syndrome is a rare autosomal dominant genetic disease resulting from various mutations in SCN5A gene concerned with ion channels in the cardiac conduction system [4]. An es timated prevalence of the disease is approximately $5: 10,000$ and the mutations are identified in $11 \%$ to $28 \%$ of patients with BS $[2,3]$. The ECG of type 1 Brugada pattem is characterized by a coved-type ST segment elevation of at least $2 \mathrm{~mm}$ in the right precordial leads (V1-V3), followed by negative $T$ waves. These patients may be asymptomatic, but they are at risk of ventricular arrhythmia and sudden death. Type 2 pattern shows a saddle-back appearance and either a positive or biphasic $T$ wave, and the type 3 pattern presents a right precordial ST elevation of $<1 \mathrm{~mm}$ of saddle-back type or coved type. [5, 6]

Establishing the diagnosis can be difficult. The characteristic of ECG is concealed in up to $30 \%$ of patients and can only be seen after pharmacological provocation [7]. For definitive diagnosis of BS, patients with spontaneous or induced type 1 ECG pattern should satisfy the one of the following clinical criteria, his tory of ventricular fibrillation or tachycardia (VT), family history of sudden cardiac death under 45 year of age, coved-type ECGs in family members, inducibility of VT with programmed electrical stimulation, syncope or nocturnal agonal respiration $[5,6]$.

If the diagnosis is made, ICD implantation can be considered for all patients with positive symptoms and for those who have inducible VT/VF during electrophysiologic study (EPS) [8]. In this case, he was asymptomatic and had a change of ECG pattern after a drug challenge. He did not undergo EPS and was not taken ICD implantation under his cardiologist's judgment.

Perioperative malignant arrhythmias can be triggered by several pharmacological and physiological factors, such as medications routinely used in anesthetic practice, electrolyte imbalances, temperature variations, postural changes, physiologicals tress and increased vagal activity [3]. According to a retrospective chart review at the Mayo clinic by Kloesel et al., they reported that some anesthetic agents, propofol, etomidate, lidocaine, and succinylcholine, were noted to havea temporal association to ST segment elevations. Therefore, anesthesiologists should pay particular attention to use appropriate drugs reported to be safer and to manage the confounding factors not to induce the cris is in a perioperative period [4].

There was a report regarding the relationship of ECG changes and sudden death in propofol infusion syndrome (PRIS) in the study of Vernooy et al., which explained that PRIS induced a Bragada ECGpattern [9]. However, clinical experience 
does not support the recommendation of avoiding bolus dosing for induction in patients with BS. Thiopental use has been described in multiple case reports without problems, while self-limited ST segment elevations were noted following etomidate administration in the review of Kloes el et al. [4].

In this case, even if propofoluse as a bolus for induction is tolerable for BS patients, we used thiopental, known to be safer, and rocuroniumas a neuromuscular blocking agent, regarding the reports that both depolarizing and nondepolarizing agents have been utilized clinically without any incidents. To antagonize neuromuscular blockade, we used sugammadex instead of cholinesterase inhibitors because it was reported as a reversal agent of choice for steroidal nondepolarizing agents, and no complication was presented with it [3].

Prior to the surgery of BS patients, anesthesiologists are required to have special vigilance. External defibrillation pads should be ready to be used before starting anesthesia, and a continuous ECG recording and monitoring of some parameters such as the bispectral index, core temperature, the degree of neuromuscular block, and arterial blood pressure are required [3]. After the surgery, the follow-up of these patients should continue in the immediate postoperative period with postoperative analgesia under continuous ECG monitoring during the first 24 hours because autonomic changes can contribute the development of tachyarrhythmia [10].

\section{CONCLUSION}

Brugada syndrome is rare, but predisposing patients to potentially fatal arrhythmias. This dangerous condition can be induced by some factors such as anesthetic agents as well as increased vagal tone, inadequate analgesia, electrolyte imbalances, and thermal variations in a perioperative period. Accordingly, anesthesiologis ts should be well aware of using the safer drugs, monitor closely, and be prepared to manage the unexpected events. The timely management can decrease the mortality and morbidity, leading to betteroutcomes.

\section{REFERENCES}

1. Brugada P, Brugada J, "Right bundle branch block, pers istent ST segment elevation and sudden cardiac death: a distinct clinical and electrocardiographic syndrome. A multicenter report" J Am Coll Cardiol. Vol.20, No.6: pp.1391-6, 1992.

2. Benito B, Brugada J, "Brugada syndrome” Rev Española Cardiol. Vol.62, No.11: pp.1297-315, 2009.

3. Dendramis G, Paleologo C, "Anesthetic and perioperativemanagement of patients with Brugada syndrome" Am J Cardiol. Vol.120: pp.1031-1036, 2017.

4. Kloesel B, Ackerman MJ, "Anesthetic management of patients with Brugada syndrome: a case series and literature review" Can J Anaesth. Vo1.58: pp.824-836, 2011.

5. Wilde AAM, Antzelevitch C, "Proposed diagnostic criteria for the Brugada syndrome. Consensus report" Eur Heart J. Circulation. Vol.106: pp. 2514-2519, 2002.

6. Antzelevitch C, Brugada P, "Brugada syndrome. Report of the second consensus conference" Eur Heart Rhythm Association. Circulation. Vol.111:pp.659-670, 2005.

7. Edge CJ, Blackman DJ, "General anes thesia in a patient with Brugada syndrome” Br J Anaesth Vol.89: pp.78891, 2002.

8. Conde R, Pereira M, "Anesthetic management of a patient with Brugada syndrome - the use of sugammadexin major abdominal surgery” Rev Bras Anestesiol. Vol.63, No.1: pp.159-162, 2013.

9. Vernooy K, Delhaas T, "Electrocardiographic changes predicting sudden death in propofol-related infusion syndrome" Heart Rhythm. Vo1.3: pp.131-7, 2006.

10. Canbay O, Erden IA, "Anesthetic management of a patient with Brugada syndrome" Pediatric Anesthesia. Vol.17: pp.1215-1227, 2007. 